\title{
ESTUDIO BIBLIOMÉTRICO DE LA PRODUCCIÓN CIENTÍFICA SOBRE LA VIOLENCIA SEXUAL EN EL CONFLICTO ARMADO, CON ÉNFASIS EN COLOMBIA *
}

\section{Bibliometric study of scientific production on conflict-related sexual violence, with emphasis in Colombia}

Liliana Rocío Chaparro Moreno**

Ana María Jiménez Pava***

Recepción: 15 de agosto de 2020. Aceptación: 23 de enero de 2021.

DOI: http://dx.doi.org/10.21017/Rev.Repub.2021.v30.a96

\section{RESUMEN}

Este artículo analiza la producción científica encontrada en Scopus y Web of Science sobre la violencia sexual relacionada con el conflicto armado a nivel general y en Colombia, hasta el año 2019. El análisis permite identificar la evolución de este campo de conocimiento, las características de las publicaciones, de la productividad de las autoras y el análisis del contenido de los principales temas de investigación.

Metodología: Análisis bibliométrico de corte exploratorio-descriptivo desarrollado a partir de tres fases: identificación de criterios y selección de palabras clave; búsqueda y sistematización de datos y análisis de los resultados.

Población: Publicaciones Scopus y Web of Science hasta 2019.

* Artículo producto de la investigación del Proyecto Fodein 2021: «Los aportes en violencia sexual de Justicia y Paz a la Justicia Especial para la Paz: un análisis jurisprudencial», Grupo de investigación socio humanística del derecho, avalado por Universidad Santo Tomás.

** Candidata al Doctorado en Ciencias Humanas y Sociales de la Universidad Nacional de Colombia. Docente de la Maestría en Defensa de los Derechos Humanos y el DIH ante Tribunales, Cortes y Organismos Internacionales de la Facultad de Derecho de la Universidad Santo Tomás (Bogotá).

Correo electrónico: lilianachaparro@usantotomas.edu.co ORCID: 0000-0002-2063-0320.

*** Magíster en Human rights and research methods de la Universidad de Essex. Docente de la Maestría en Defensa de los Derechos Humanos y el DIH ante Tribunales, Cortes y Organismos Internacionales de la Facultad de Derecho de la Universidad Santo Tomás (Bogotá).

Correo electrónico: anajimenezp@usantotomas.edu.co ORCID: https:/ /orcid.org/00000002-8504-4680. 

en el conflicto armado, con énfasis en Colombia

Mediciones principales: Crecimiento, autores con más publicaciones, autores con más citaciones, publicaciones por año, áreas de investigación, tipos de documento, países de mayor producción académica.

Criterio de inclusión: Publicaciones indexadas en Scopus y Web of Science hasta el año 2019.

Resultados: Las publicaciones reportadas por ambas plataformas se relacionan con el campo de las ciencias sociales y del derecho, siendo los principales documentos publicados los artículos en revistas académicas. La mayor producción académica está disponible en idioma inglés. La mayoría son autoras individuales, aunque en el caso de la producción sobre Colombia también se reportan autores institucionales. Es mínimo el reporte de la producción académica de organizaciones sociales que han estudiado el tema.

Palabras clave: violencia sexual, conflicto armado, guerra, Colombia.

\section{ABSTRACT}

This article analyzes the academic production on sexual violence related to the armed conflict at global level and in Colombia, reported in Scopus and Web of Science, until the year 2019. This analysis allows identifying the evolution of the issue, the characteristics of the publications, the productivity of the authors and the main research topics.

Methods: Exploratory-descriptive bibliometric analysis developed in three phases: identification of criteria and selection of keywords; search and systematization of data and results analysis.

Publication: Scopus and Web of Science until 2019.

Sample: Growth, authors with more publications, authors with more citations, publications per year, research areas, document types, countries with the highest academic production.

Results: The publications reported by both platforms are related to the fields of social sciences and law. The main documents published are articles in academic journals. The largest academic output is available in English. Most are individual authors, although institutional authors are also reported in the case of the academic production on Colombia. The report of the academic production of social organizations that have studied the subject is minimal.

Keywords: sexual violence, armed conflict, war, Colombia.

\section{INTRODUCCIÓN}

Los estudios sobre la violencia sexual asociada al conflicto armado han tendido al aumento en la literatura internacional (Engle, 2020, pos. 1005), en parte 
gracias a la atención que le han dado a la problemática los organismos internacionales de derechos humanos.

Al margen de ello, la violencia sexual en las guerras ocupa en la actualidad un lugar central en los estudios sobre los conflictos armados, la justicia transicional y los derechos de las víctimas. No obstante, no existen análisis bibliométricos específicos sobre la temática que contribuyan a la identificación del campo de conocimiento, aunque sí existen algunos referidos a la cuestión de la violencia contra las mujeres (Judith Velasco et al., s. f.; Brilhante et al., 2016) y las mujeres, la paz y la seguridad (Palomo et al., 2017).

Colombia ha sido un caso relevante como escenario de estudio y producción de literatura sobre la violencia sexual en tiempos de guerra, principalmente en el ámbito de los organismos internacionales que vigilan la situación de derechos humanos, debido al conflicto armado interno que enfrenta el país hace más de seis décadas ${ }^{1}$. Sin embargo, el tema no ha tenido igual relevancia en la producción académica y solo hasta hace muy poco, ha recibido una mayor atención como se explica más adelante. Esto puede deberse, en parte, a que la investigación en este campo estuvo mayormente centrada en los casos de Ruanda, Yugoslavia y Sierra Leona, países que enfrentaron conflictos armados de gran escala y en los cuales se llevaron a cabo procesos de paz y se instalaron mecanismos de justicia transicional. También porque el caso colombiano resulta desde muchos aspectos sui generis, en términos del tipo conflicto armado, siendo este el más largo de América Latina; porque se trata de un Estado no catalogado como frágil y por el contrario con un aparato institucional cada vez más fuerte (Gutiérrez Sanín, 2014) y, especialmente, debido al desconocimiento de las dinámicas de la guerra adoptadas por los actores armados.

En este contexto, el presente estudio presenta un análisis bibliométrico sobre la literatura internacional existente en Scopus y Web of Science sobre la violencia sexual relacionada con el conflicto armado en Colombia.

1 Entre 1981 y el 2014 los órganos del Sistema Interamericano de Derechos Humanos y de las Naciones Unidas se pronunciaron sobre la situación colombiana en al menos 40 oportunidades y en al menos 23 informes hicieron referencia a la cuestión de la violencia sexual en el conflicto armado. Los informes más notorios sobre el tema son Relatora Especial sobre la violencia contra la mujer, sus causas y consecuencias, ONU, 2002; Comisión Interamericana de Derechos Humanos, 2006; Corte Penal Internacional, 2012, y Office of Special Representative of the Secretary-General on Sexual Violence in Conflict, 2011. 


\section{MATERIALES Y MÉTODOS}

El estudio correspondió a un análisis bibliométrico, cuyo objeto fueron las publicaciones Scopus y Web of Science sobre violencia sexual relacionada con el conflicto armado en general y para el caso colombiano. Se utilizaron las bases de datos Harzing's Publish or Perish (POP) (versión 7.21.2811.7445), VosViewer (versión 1.6.15), Vantage Point (versión 12.0) y Zotero (versión 5.0.88) y el programa de Microsoft Excel. La búsqueda se realizó entre el 10 y el 30 de junio de 2020, empleando las siguientes ecuaciones de búsqueda:

Tabla 1. Bases de datos consultadas para el análisis infométrico y ecuaciones de búsqueda.

\begin{tabular}{|l|l|}
\hline Fuente & Búsqueda \\
\hline Scopus & $\begin{array}{l}\text { Ecuación de búsqueda utilizada: «conflict-related sexual } \\
\text { violence»; «sexual violence» and war; «sexual violence» and } \\
\text { «armed conflict»; «violencia sexual» and «conflicto armado». } \\
\text { Posteriormente se tuvo como parámetro de inclusión } \\
\text { aquellos documentos que contuvieran la palabra «Colombia» } \\
\text { y los documentos duplicados fueron fusionados. }\end{array}$ \\
\hline Web of Science & $\begin{array}{l}\text { Ecuación de búsqueda utilizada: «conflict-related sexual } \\
\text { violence»; «sexual violence» and war; «sexual violence" and } \\
\text { «armed conflict»; «violencia sexual" and «conflicto armado». } \\
\text { Frente a cada resultado se delimitó la búsqueda al criterio } \\
\text { «Colombia». Los documentos duplicados fueron fusionados. }\end{array}$ \\
\hline
\end{tabular}

Dentro de los criterios de inclusión se consideraron todos los documentos Scopus y Web of Science hasta el año 2019. Las variables analizadas en este artículo están señaladas en la tabla 2.

Los objetivos e indicadores tomados en cuenta se basaron fundamentalmente en el estudio de Palomo et al. (2017) y fueron modificados para los efectos de este análisis. En la tabla 3 se presentan los objetivos, las preguntas y los indicadores tenidos en cuenta.

\section{RESULTADOS}

$\mathrm{Al}$ analizar las categorías descritas y fusionar la información duplicada en la plataforma Scopus, se encontró un total de 836 resultados. De ellos, 72 contienen el vocablo «Colombia» como refinador en la búsqueda, arrojando documentos desde el año 2006. Para el caso de la plataforma Web of 
Tabla 2. Variables analizadas según fuente consultada.

\begin{tabular}{|c|c|}
\hline Fuente & Variables \\
\hline Scopus & $\begin{array}{l}\text { - Información de citación: Author(s), Author(s) ID, } \\
\text { Document title, Year, EID, Source title, volume, issue, } \\
\text { pages, Citation count, Source \& document type, } \\
\text { Publication Stage, DOI, Access Type. } \\
\text { - Información bibliográfica: Affiliations, Serial } \\
\text { identifiers (e.g. ISSN), PubMed ID, Publisher, } \\
\text { Editor(s), Language of original document, } \\
\text { Correspondence address, Abbreviated source title. } \\
\text { - Resumen y palabras clave: Abstract, Author } \\
\text { keywords, Index keywords. } \\
\text { - Detalle de fondos: Number, Acronym, Sponsor, } \\
\text { Funding text. } \\
\text { Otra información: Tradenames \& manufacturers, } \\
\text { Accession numbers \& chemicals, Conference } \\
\text { information, Include references. }\end{array}$ \\
\hline Web of Science & $\begin{array}{l}\text { Autor(es)/Editor(es), Abstract, Direcciones, ISSN/ISBN, } \\
\text { Número IDS, Información de financiación, ID de } \\
\text { PubMed, Título, Referencias citadas, Veces citado, } \\
\text { Número de referencias citadas, Idioma, Número de } \\
\text { acceso, Acceso Abierto, Fuente, Tipo de documento, } \\
\text { Palabras clave, Abrev. de fuente, Categorías de Web of } \\
\text { Science, Identificadores de autores, Artículo popular, } \\
\text { Información sobre la conferencia, Patrocinadores de la } \\
\text { conferencia, Información sobre la editorial, Número de } \\
\text { páginas, Áreas de investigación, Conteo de uso, Muy } \\
\text { citado. }\end{array}$ \\
\hline
\end{tabular}

Science, encontramos un total de 606 resultados en búsqueda por tema, de los cuales tan solo 18 hacen referencia específica a Colombia, como se ilustra en la tabla 4.

Como se observa en la tabla 4 , los criterios de búsqueda en idioma inglés son los que arrojan más resultados, mientras que aquel en idioma español presenta tan solo 5 documentos reportados en Scopus y ninguno en Web of Science. Esto tiene impactos al momento de articular el conocimiento desarrollado en la literatura académica internacional con la producción local, puesto que no necesariamente existe una conexión entre ambas esferas de conocimiento debido, en parte, al acceso al idioma en que se producen. 

en el conflicto armado, con énfasis en Colombia

Tabla 3. Objetivos, preguntas e indicadores.

\begin{tabular}{|c|c|c|}
\hline Objetivo & Pregunta & Indicador \\
\hline $\begin{array}{l}\text { A. Evolución del } \\
\text { área }\end{array}$ & $\begin{array}{l}\text { ¿Cuándo se publicaron } \\
\text { los primeros } \\
\text { documentos? } \\
\text { ¿Cuál es la evolución de } \\
\text { la producción a lo largo } \\
\text { del tiempo? }\end{array}$ & $\begin{array}{l}\text { 1. Número y años de } \\
\text { publicación de los primeros } \\
\text { documentos. } \\
\text { 2. Número y años de } \\
\text { publicación hasta } 2019 \text {. }\end{array}$ \\
\hline $\begin{array}{l}\text { B. Características } \\
\text { de las } \\
\text { publicaciones }\end{array}$ & $\begin{array}{l}\text { ¿Cómo se agrupan los } \\
\text { documentos según el } \\
\text { área de investigación? } \\
\text { ¿Cuáles son las revistas } \\
\text { que publican artículos } \\
\text { en violencia sexual en } \\
\text { conflicto armado, en } \\
\text { particular en el caso } \\
\text { colombiano? } \\
\text { ¿Qué tipo de } \\
\text { documentos son los más } \\
\text { publicados? }\end{array}$ & $\begin{array}{l}\text { 1. Distribución de artículos } \\
\text { basada en áreas de } \\
\text { investigación. } \\
\text { 2. Número y lista de revistas } \\
\text { más relevantes en función } \\
\text { del número de artículos } \\
\text { publicados. } \\
\text { 3. Caracterización de los } \\
\text { documentos según tipo. }\end{array}$ \\
\hline $\begin{array}{l}\text { C. Características } \\
\text { y } \\
\text { productividad } \\
\text { de autores }\end{array}$ & $\begin{array}{l}\text { ¿Quiénes son los autores } \\
\text { más prolíficos? } \\
\text { ¿Cuál es el nivel de } \\
\text { colaboración entre } \\
\text { autores? } \\
\text { ¿Cuáles son los países } \\
\text { de mayor producción } \\
\text { académica en el tema? }\end{array}$ & $\begin{array}{l}\text { 1. Lista de autores más } \\
\text { relevantes. } \\
\text { 2. Índice de colaboración. } \\
\text { 3. Lista de países con mayor } \\
\text { producción. }\end{array}$ \\
\hline $\begin{array}{l}\text { D. Análisis de } \\
\text { contenido: } \\
\text { temas de } \\
\text { investigación }\end{array}$ & $\begin{array}{l}\text { ¿Cuáles son los temas } \\
\text { cubiertos? } \\
\text { ¿Cuáles son las palabras } \\
\text { clave que describen la } \\
\text { investigación en el } \\
\text { campo? }\end{array}$ & $\begin{array}{l}\text { 1. Lista de temas e importancia } \\
\text { de cada uno. } \\
\text { 2. Nube de palabras clave. }\end{array}$ \\
\hline
\end{tabular}

Fuente: Elaboración propia basada en Palomo et al., 2017.

El criterio «sexual violence» AND war es el que más resultados arroja en ambas bases de datos, por encima de otros que directamente asocian la alocución a conflicto armado, lo cual evidencia que en la literatura científica internacional la guerra es aún el campo de producción más prolífico. 
Tabla 4. Resultados por criterio de búsqueda y plataforma.

\begin{tabular}{|l|l|l|}
\hline \multicolumn{1}{|c|}{ Criterio de búsqueda } & Scopus & $\begin{array}{l}\text { Web of } \\
\text { Science }\end{array}$ \\
\hline «Sexual violence» AND war & 683 & 500 \\
\hline «Sexual violence» AND «armed conflict» & 230 & 160 \\
\hline «-related sexual violence» & 96 & 76 \\
\hline «Violencia sexual» AND «Conflicto armado» & 5 & 0 \\
\hline Total de resultados (luego de fusionar los duplicados) & 836 & 606 \\
\hline
\end{tabular}

\section{A. Evolución del área}

De acuerdo con el gráfico 1, de los 836 resultados identificados en la plataforma Scopus en materia de violencia sexual en el conflicto armado, se observa que aunque el documento más antiguo data de 1988, no hay nuevas publicaciones sobre el tema hasta el año 1993, y es solo hasta el 2005 que se identifica un aumento de las publicaciones, superando los 11 números por año y reportando un aumento sostenido hasta 2019, siendo el año de mayor producción el 2017 con 89 documentos.

Para el caso colombiano, en cambio, el primer documento que da cuenta de la violencia sexual en el conflicto armado es registrado por Scopus en el año 2006, más de 18 años después del primer documento reportado en el tema bajo el criterio de búsqueda general. A partir del año 2008 de manera sostenida se ve un incremento en la producción académica, siendo el año 2019 el de mayor publicación con 12 documentos.

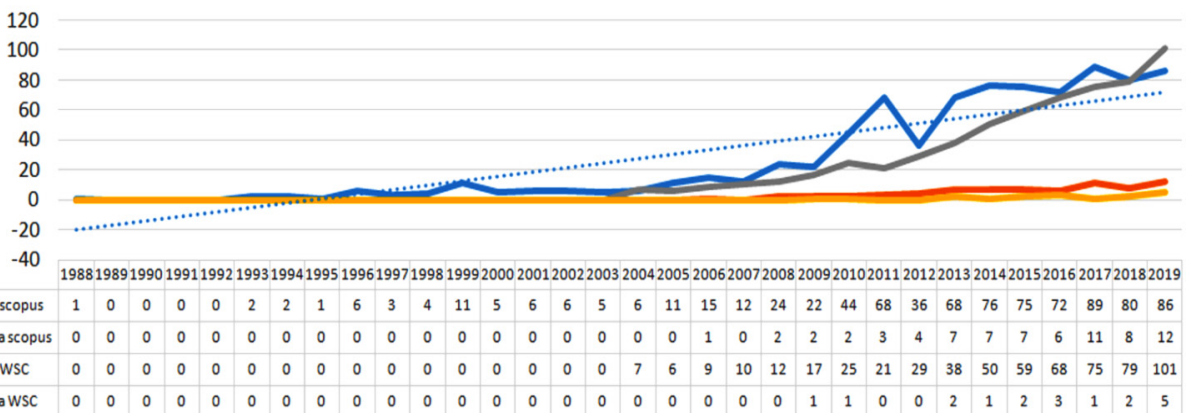

Gráfico 1. Evolución por año y plataforma de la producción académica en general y en Colombia.

Fuente: Construcción propia a partir de los resultados de Scopus y Web of Science. 

en el conflicto armado, con énfasis en Colombia

En el caso de la plataforma Web of Science se encontró que la publicación más antigua corresponde al año 2004 y que los años posteriores se mantuvo la producción académica, alcanzando en el 2019 el más alto nivel con 101 documentos. En lo que respecta al caso colombiano, en el año 2009 se reportó la primera publicación, pero solo hasta el año 2014 se evidencia una producción académica sostenida.

A partir de la información reportada en ambas plataformas es posible afirmar que la diferencia numérica entre ambas es de 230 documentos, diferencia que tiene relación con el inicio de los años de publicación. Mientras que en Scopus la publicación periódica en el tema inició en el año 1993, en Web of Science este proceso se dio solo una década después, en el año 2004. Sin embargo, en lo que se refiere al caso colombiano, la diferencia no es sustancialmente notoria frente a los años de publicación: mientras que en Scopus se inicia en 2006, en Web of Science comienza en 2009.

Entre los años 2005 y 2006 se identifica un crecimiento importante de la producción académica reportada en ambas plataformas, lo cual coincide con el desarrollo de la Agenda de Mujer, Paz y Seguridad (Theidon, 2016; Engle, 2020; Henry, 2014), que se inauguró con la Resolución 1325 de 2000 del Consejo de Seguridad de las Naciones Unidas, que estaba puesta en marcha en varios lugares del mundo. Esta Agenda estableció la necesidad de adoptar medidas para asegurar el aumento de la representación de las mujeres en los procesos de paz en el mundo y proteger a las mujeres y niñas de los efectos de las guerras, en particular «la violación y otras formas de abusos sexuales».

A partir del año 2008, la producción académica se incrementó, pasando para el caso de Scopus de 12 publicaciones en 2007 a 24 documentos en 2008, y para Web of Science de 10 en 2007 a 12 documentos en 2008. Esto coincide con la expedición de la Resolución 1820 de 2008 del Consejo de Seguridad de las Naciones Unidas, que centralizó dentro de la agenda de la organización la cuestión de la violencia sexual en asocio a temas de paz y seguridad internacional. Para el año 2011 la producción académica reportada en Scopus experimentó un crecimiento vertiginoso aumentando casi tres veces el registro de documentos en relación a la cifra del año 2009. No obstante, el año siguiente se dio un descenso dramático de las publicaciones científicas registradas por Scopus, de casi la mitad de documentos registrados en 2011.

En el año 2013 se identifica nuevamente un crecimiento de la producción académica en el tema de la violencia sexual tanto en Scopus como en Web of Science que se mantiene en aumento hasta el año 2019. 


\section{B. Características de las publicaciones}

$\mathrm{Al}$ analizar las publicaciones académicas registradas el área de investigación de la violencia sexual asociada al conflicto armado (gráfico 2), se encontró que en el caso de Scopus la mayoría de documentos se enmarcan en el área de conocimiento de las ciencias sociales $(47,4 \%)$, seguido de medicina $(20,2 \%)$, artes, humanidades $(16,4 \%)$ y psicología $(7 \%)$.

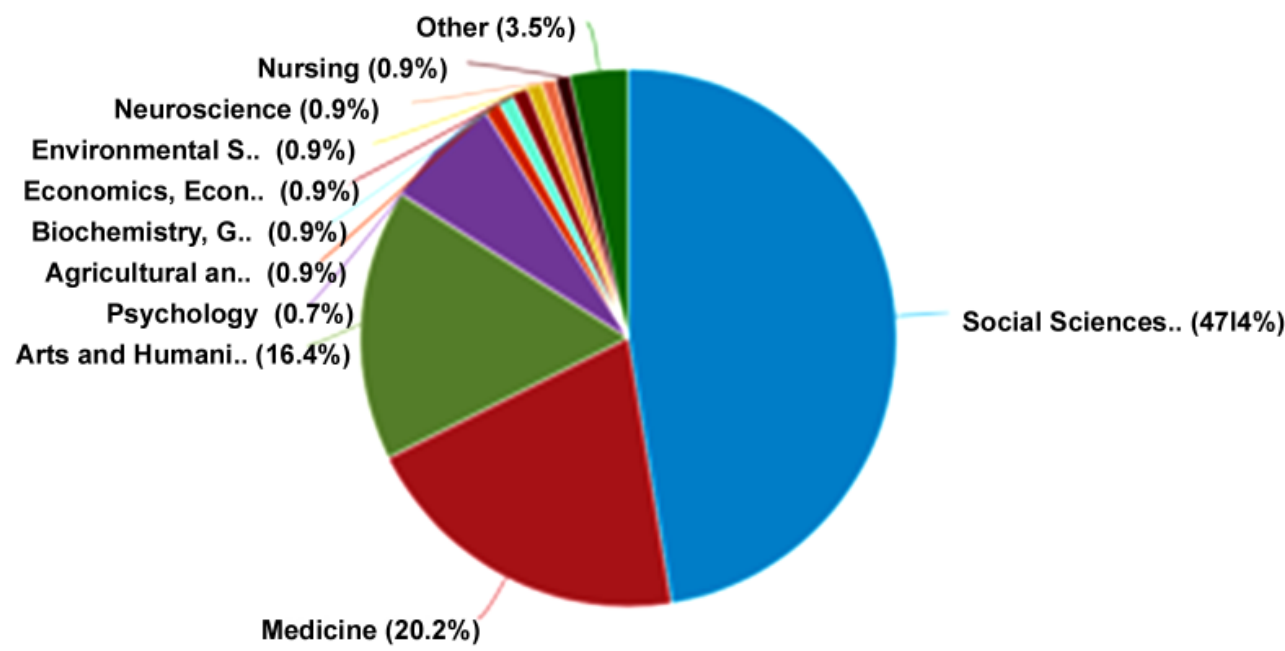

Gráfico 2. Área de investigación de violencia sexual en conflicto armado - Scopus. Fuente: Scopus

Lo mismo ocurre en el caso de las publicaciones específicas sobre el tema de la violencia sexual asociada al conflicto armado en Colombia (gráfico 3), siendo incluso mayor la distribución en las ciencias sociales $(60,6 \%)$ que en la medicina $(17 \%)$. No obstante, a diferencia de las publicaciones no relacionadas con Colombia analizadas previamente, los campos de estudio del tema se reducen a ocho, no reportándose documentos en el área de la enfermería, la neurociencia o el medio ambiente.

Tratándose de la información reportada para la violencia sexual en general en Web of Science (gráfico 4), la mayoría de publicaciones se concentran en las áreas de derecho (con 196 publicaciones que equivalen al 32,3\%), las relaciones internacionales (con 109 documentos que representan el 17,9\%), los estudios sobre mujer (con 94 documentos que equivalen al 15,5\%), el medio ambiente y la salud ocupacional (60 documentos que representan el 9,9\%), entre otras disciplinas. 


\section{Multidiscipli.na.. (1.1\%)}

Biochemistry, G.. $(1,1 \%)$

Agricultural an.. $(1,1 \%)$

Business, Manag.. (2.1\%)

Psychology $(5.3 \%)$

Arts and Humani.. (11.7\%)

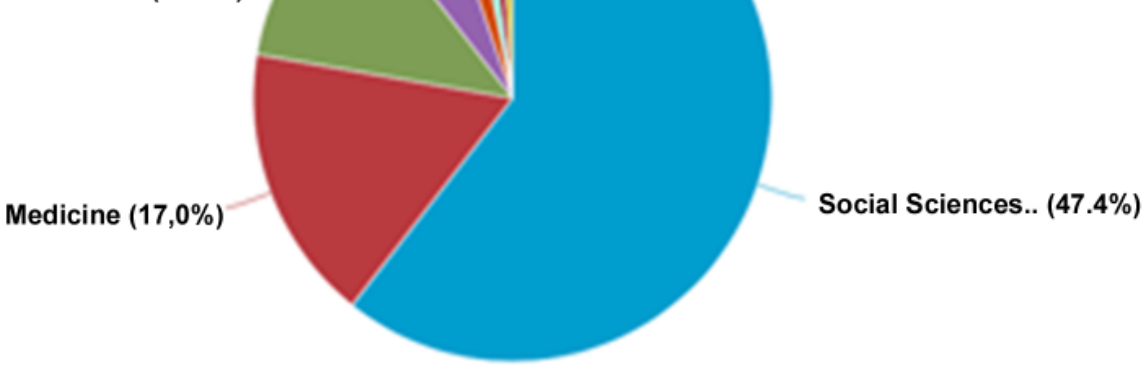

Gráfico 3. Área de investigación de violencia sexual en conflicto armado en Colombia - Scopus. Fuente: Scopus.
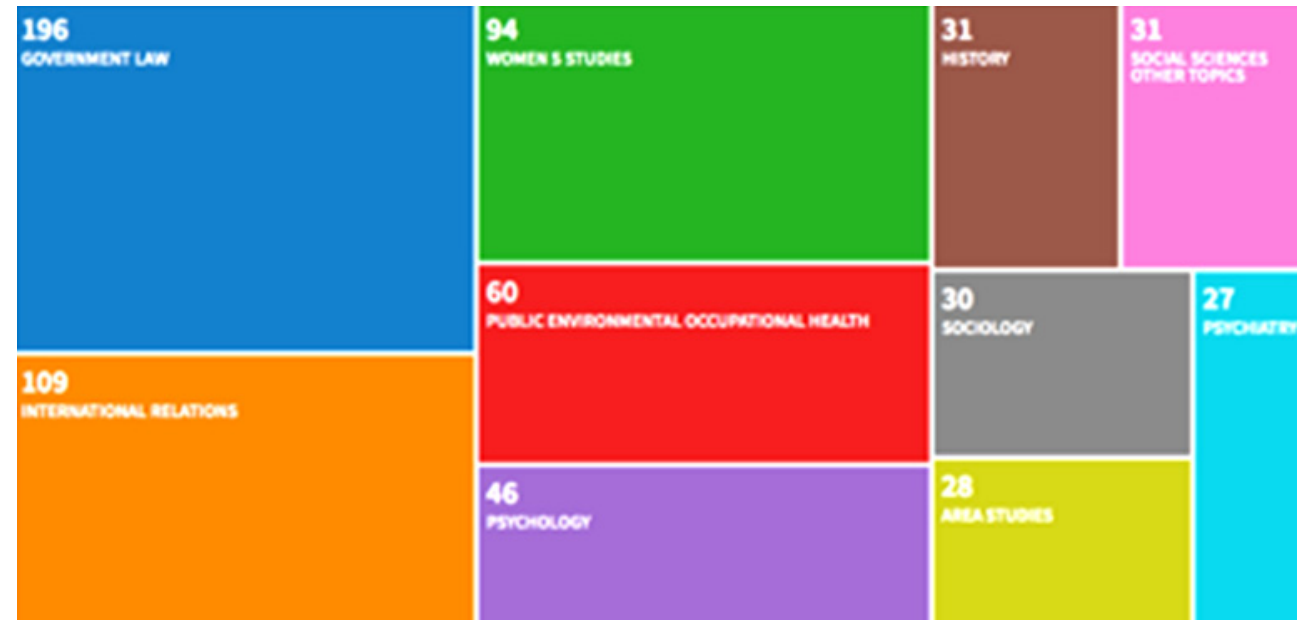

Gráfico 4. Área de investigación de violencia sexual en conflicto armado - Web of Science. Fuente: Web of Science.

Los resultados reportados en Web of Science en materia de la violencia sexual en Colombia (gráfico 5) dan cuenta de la prevalencia de las áreas del derecho $(32,3 \%)$ y las relaciones internacionales $(17,9 \%)$, lo cual resulta significativo, pues denota un desplazamiento de otras áreas como la medicina y la psicología las cuales han liderado a nivel general el estudio de este campo. Lo mismo ocurre en el caso colombiano (61,1\% para derecho y 33,3\% para relaciones internacionales). 


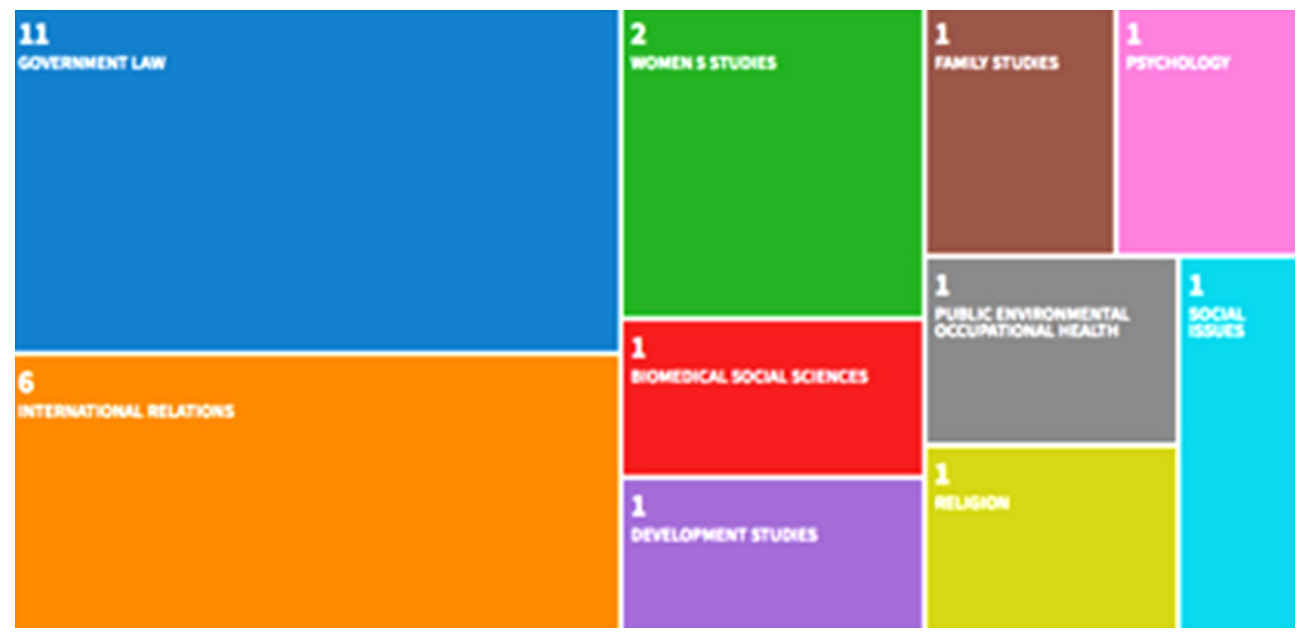

Gráfico 5. Área de investigación de violencia sexual en conflicto armado en Colombia - Web of Science. Fuente: Web of Science.

De otro lado, en relación con la tipología de documentos (tabla 5) se identifica que en ambas plataformas de búsqueda los artículos en revista científica (542 en Scopus y 491 en Web of Cience) representan la mayor cantidad del total de documentos indexados y si bien los capítulos de libro les siguen en orden, llama la atención que en Scopus se reportan 112 documentos de este tipo mientras que en Web of Science solo se reportan 2, lo cual evidencia una diferencia significativa si se tiene en cuenta que el primero reporta 40 libros sobre el tema mientras que el segundo no reporta ninguno.

En relación con las revistas que más publican artículos sobre el tema (tabla 6), Scopus reporta que Lancet - revista médica británica - lidera la producción académica en el tema con 18 publicaciones que representan 2,1\% del total de

Tabla 5. Tipo de documento publicado.

\begin{tabular}{|l|c|c|}
\hline & Scopus & Web of Science \\
\hline Artículo & 542 & 491 \\
\hline Capítulo de libro & 112 & 2 \\
\hline Libro & 40 & 0 \\
\hline Reseña & 97 & 90 \\
\hline Otros & 45 & 36 \\
\hline
\end{tabular}

Fuente: Scopus y Web of Science. 

en el conflicto armado, con énfasis en Colombia

Tabla 6. Revistas con mayor número de publicaciones.

\begin{tabular}{|c|l|c|l|c|l|c|l|c|}
\hline \multicolumn{1}{|c|}{$\begin{array}{c}\text { Scopus } \\
\text { general }\end{array}$} & Núm. & $\begin{array}{c}\text { Scopus } \\
\text { Colombia }\end{array}$ & Núm. & \multicolumn{1}{|c|}{$\begin{array}{c}\text { WSC } \\
\text { general }\end{array}$} & Núm. & $\begin{array}{l}\text { WSC } \\
\text { Colombia }\end{array}$ & Núm. \\
\hline 1. & Lancet & 18 & $\begin{array}{l}\text { International } \\
\text { Review of } \\
\text { The Red } \\
\text { Cross }\end{array}$ & 3 & $\begin{array}{l}\text { International } \\
\text { Feminist } \\
\text { Journal of } \\
\text { Politics }\end{array}$ & 22 & $\begin{array}{l}\text { International } \\
\text { Journal of } \\
\text { Transitional } \\
\text { Justice }\end{array}$ & 8 \\
\hline 2. & $\begin{array}{l}\text { International } \\
\text { Feminist } \\
\text { Journal of } \\
\text { Politics }\end{array}$ & 16 & $\begin{array}{l}\text { Journal of } \\
\text { Peace } \\
\text { Research }\end{array}$ & 3 & $\begin{array}{l}\text { International } \\
\text { Review of } \\
\text { The Red } \\
\text { Cross }\end{array}$ & 14 & $\begin{array}{l}\text { Conflict } \\
\text { And Health }\end{array}$ & 1 \\
\hline 3. & $\begin{array}{l}\text { International } \\
\text { Review of The } \\
\text { Red Cross }\end{array}$ & 15 & $\begin{array}{l}\text { Revista } \\
\text { Colombiana } \\
\text { de } \\
\text { Psiquiatría }\end{array}$ & 3 & $\begin{array}{l}\text { Journal Of } \\
\text { Peace } \\
\text { Research }\end{array}$ & 13 & $\begin{array}{l}\text { Culture } \\
\text { Health } \\
\text { Sexuality }\end{array}$ & 1 \\
\hline $\mathbf{4 .}$ & $\begin{array}{l}\text { Reproductive } \\
\text { Health } \\
\text { Matters }\end{array}$ & 12 & $\begin{array}{l}\text { Análisis } \\
\text { Político }\end{array}$ & 2 & $\begin{array}{l}\text { International } \\
\text { Journal of } \\
\text { Transitional } \\
\text { Justice }\end{array}$ & 12 & $\begin{array}{l}\text { Derecho y } \\
\text { Ciencias } \\
\text { Sociales }\end{array}$ & 1 \\
\hline 5. & $\begin{array}{l}\text { Conflict And } \\
\text { Health }\end{array}$ & 11 & $\begin{array}{l}\text { Conflict And } \\
\text { Health }\end{array}$ & 2 & $\begin{array}{l}\text { Violence } \\
\text { Against } \\
\text { Women }\end{array}$ & 11 & Dixi & 1 \\
\hline
\end{tabular}

Fuente: Construcción propia a partir de Scopus y Web of Science.

documentos. En el caso de Web of Science el primer lugar lo ocupa la revista International Feminist Journal of Politics con 22 artículos equivalentes al 3,6\% del total de documentos.

En cuanto a las revistas específicas sobre la violencia sexual en el conflicto armado en Colombia (tabla 6) Scopus reporta la revista International Review Of The Red Cross que cuenta con 3 documentos, el Journal Of Peace Research (con 3 documentos) y la Revista Colombiana de Psiquiatría (con 3 documentos). Por su parte, las publicaciones en Web of Science son lideradas por el International Journal of Transitional Justice con 8 documentos, seguido de la Revista Conflict And Health con 1.

Como se observa en la tabla anterior, la mayoría de revistas que publican sobre violencia sexual en conflictos armados en general y de manera particular sobre Colombia, se relacionan con estudios feministas (International Feminist Journal Of Politics), asuntos humanitarios (International Review of the Red Cross), de justicia transicional y paz (Journal of Peace Research, International Journal of Transitional Justice) y de ciencia (Lancet, Revista Colombiana de Psiquiatría, Conflict And Health). 


\section{Características y productividad de autores}

En lo referente a las autoras con mayor productividad académica en el tema (tabla 7), se encuentran Scott, Bartels, Clark, Mukwege y Glass, de acuerdo con Scopus. Por su parte, las publicaciones reportadas por Web of Science son lideradas por Clark (10 documentos), seguida de Wood, Cohen, Nordas y Stark. En relación con el caso colombiano en específico, se identifican True, seguida de Wood, Clark, Davies y Glass, quienes tienen la mayor cantidad de artículos en el tema según Scopus. En Web of Science si bien se reportan 18 publicaciones y 41 autoras, ninguna supera una publicación.

Tabla 7. Autores y países con mayor producción en general y para el caso Colombia.

\begin{tabular}{|c|c|c|c|c|}
\hline & \multicolumn{2}{|c|}{$\begin{array}{l}\text { Violencia sexual en conflicto en } \\
\text { general }\end{array}$} & \multicolumn{2}{|c|}{$\begin{array}{l}\text { Violencia sexual en conflicto en } \\
\text { Colombia }\end{array}$} \\
\hline & $\begin{array}{l}\text { Autores con } \\
\text { mayor } \\
\text { producción }\end{array}$ & $\begin{array}{l}\text { Países de origen } \\
\text { de las } \\
\text { publicaciones }\end{array}$ & $\begin{array}{l}\text { Autores con } \\
\text { mayor } \\
\text { producción }\end{array}$ & $\begin{array}{l}\text { Países de origen } \\
\text { de las } \\
\text { publicaciones }\end{array}$ \\
\hline Scopus & $\begin{array}{l}\text { Scott, J.: } 10 \\
\text { Bartels, S.: } 9 \\
\text { Clark, J.N.: } 9 \\
\text { Mukwege, d.: } 9 \\
\text { Glass, N.: } 8\end{array}$ & $\begin{array}{l}\text { Estados Unidos: } \\
291 \\
\text { Reino Unido: } 145 \\
\text { Canadá: } 61 \\
\text { Australia: } 53 \\
\text { Alemania: } 38\end{array}$ & $\begin{array}{l}\text { True, J.: } 4 \\
\text { Wood, E.J.: } 4 \\
\text { Clark, J.N.: } 3 \\
\text { Davies, S.E.: } 3 \\
\text { Glass, N.: } 3\end{array}$ & $\begin{array}{l}\text { Estados Unidos: } \\
26 \\
\text { Colombia: } 13 \\
\text { Reino Unido: } 13 \\
\text { Australia: } 9 \\
\text { Canadá: } 5\end{array}$ \\
\hline $\begin{array}{l}\text { Web of } \\
\text { Science }\end{array}$ & $\begin{array}{l}\text { Clark, J.N.: } 10 \\
\text { Wood, E.J.: } 8 \\
\text { Cohen, D.K.: } 6 \\
\text { Nordas, R.:6 } \\
\text { Stark, 1.: } 6\end{array}$ & $\begin{array}{l}\text { Estados Unidos: } \\
217 \\
\text { Reino Unido: } 114 \\
\text { Canadá: } 50 \\
\text { Australia: } 40 \\
\text { Alemania: } 31\end{array}$ & $\begin{array}{l}\text { Del total de } 18 \\
\text { publicaciones se } \\
\text { contabilizan } 41 \\
\text { autores y } \\
\text { autoras, sin que } \\
\text { cada uno tenga } \\
\text { más de una } \\
\text { publicación. }\end{array}$ & $\begin{array}{l}\text { Colombia: } 12 \\
\text { Estados Unidos: } \\
4 \\
\text { Reino Unido: } 2 \\
\text { España: } 2 \\
\text { Australia: } 1\end{array}$ \\
\hline
\end{tabular}

Fuente: Construcción propia a partir de Scopus y Web of Science.

Al identificar las cocitaciones de la producción de Scopus frente a la violencia sexual en conflictos en general a través del programa Vosviewer, teniendo como unidad de análisis los autores citados en un mínimo de 80 citaciones por autor, encontramos que hay 17 que superan dicho umbral, como se observa en la tabla 8. 
Tabla 8. Autoras con umbral de citación de mínimo 80 en Scopus - violencia sexual en general.

\begin{tabular}{|l|c|}
\hline Autora & Citaciones \\
\hline Wood, E.J. & 224 \\
\hline Cohen, D.K. & 203 \\
\hline Stern, M. & 203 \\
\hline Enloe, C. & 181 \\
\hline Brownmiller, S. & 158 \\
\hline Scott, J. & 125 \\
\hline Johnson, K. & 114 \\
\hline Askin, K.D. & 99 \\
\hline Baaz, M.E. & 98 \\
\hline Sivakumaran, S. & 98 \\
\hline True, J. & 88 \\
\hline Copelon, R. & 87 \\
\hline Betancourt, T.S. & 87 \\
\hline Seifert, R. & 84 \\
\hline Asher, J. & 83 \\
\hline Charlesworth, H. & 83 \\
\hline Mazurana, D. & 82 \\
\hline
\end{tabular}

Fuente: Vosviewer a partir de información de Scopus.

Al ver esta información de manera gráfica se identifica con claridad que Wood, Cohen y Stern, pese a no estar en el listado de las autoras con mayor producción académica en el campo de la violencia sexual en conflictos armados, son las que tienen un mayor nivel de cocitación. Gráfico 6.

Respecto de las publicaciones específicas sobre Colombia (tabla 9), los 72 resultados arrojados por Scopus teniendo en cuenta como umbral de búsqueda autores con un mínimo de 20 cocitaciones, se encuentran 10 autoras lideradas por Wood con 57 documentos, seguida por Cohen con 46, Stern con 32 y True con 30 , entre otros autores y autoras. 


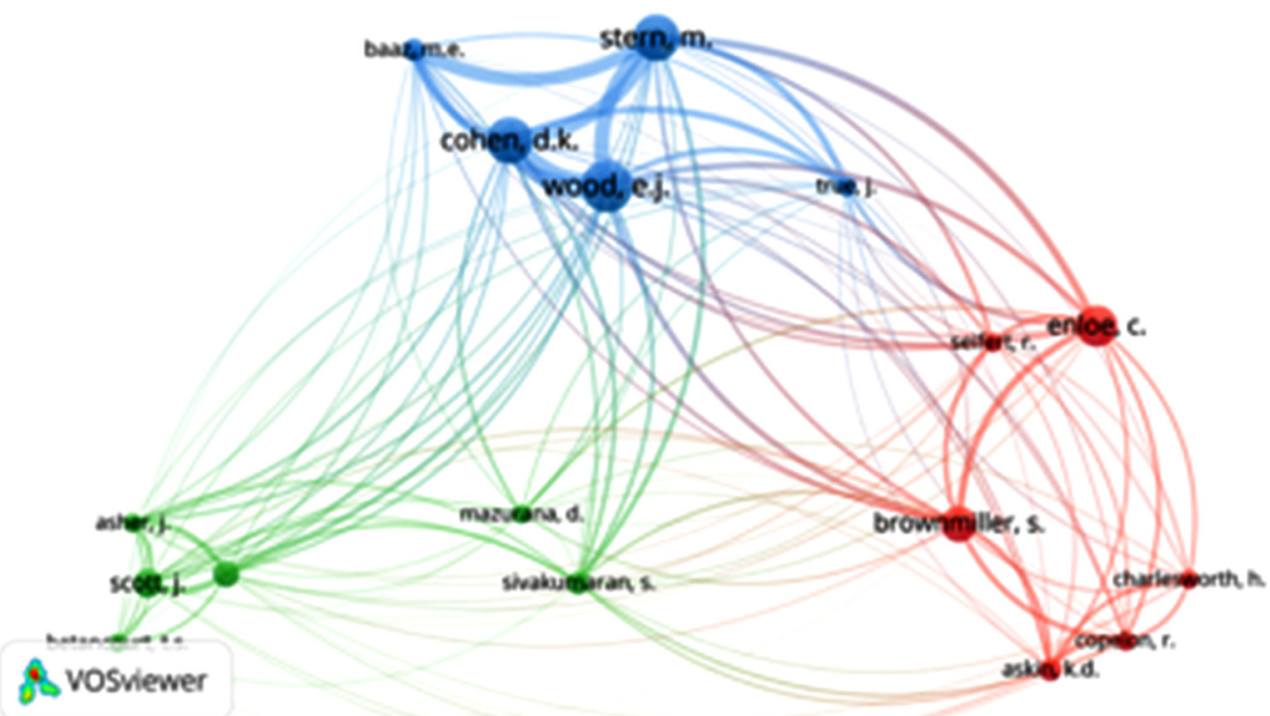

Gráfico 6. Cocitación Scopus violencia sexual en general - mínimo de citación 80 Fuente: Vosviewer a partir de información de Scopus,

Tabla 9. Autoras con umbral de citación de mínimo 10 en Scopus - violencia sexual en Colombia.

\begin{tabular}{|l|c|}
\hline Autora & Citaciones \\
\hline Wood, E.J. & 57 \\
\hline Cohen, D.K. & 46 \\
\hline Stern, M. & 32 \\
\hline True, J. & 30 \\
\hline Theidon, K. & 26 \\
\hline Scott, J. & 25 \\
\hline Clark, J.N. & 22 \\
\hline Fionnuala Ni, A. & 22 \\
\hline Green, A.H. & 21 \\
\hline Baaz, M.E. & 20 \\
\hline
\end{tabular}

Fuente: Vosviewer a partir de información de Scopus.

Del listado anterior, únicamente Wood y True se encuentran en el listado de las autoras con mayor producción sobre la temática de violencia sexual en el caso colombiano, pero destacan Cohen, Stern y Theidon como otras autoras relevantes en el campo (gráfico 7). 

en el conflicto armado, con énfasis en Colombia

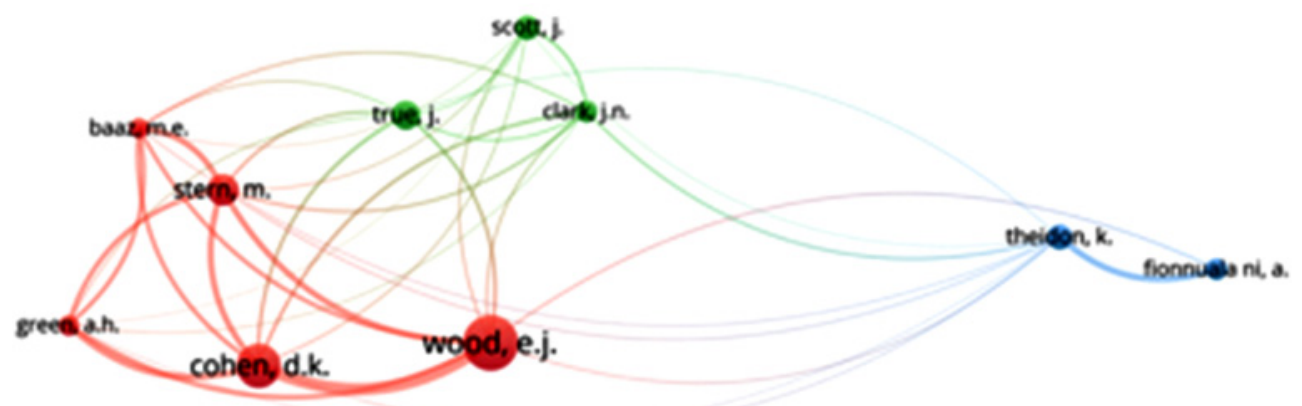

Gráfico 7. Cocitación Scopus violencia sexual en Colombia - mínimo de citación 10 Fuente: Vosviewer a partir de información de Scopus.

En el caso de Web of Science al analizar los resultados en el programa Vosviewer (tabla 10) se encuentra que, teniendo como umbral 80 citaciones de un autor mismo autor en materia de violencia sexual en general, solo 8 lo superan, coincidiendo casi en su totalidad con las autoras más relevantes reportadas por Scopus.

Tabla 10. Autoras con umbral de citación de mínimo 80 en Web of Science - violencia sexual en general

\begin{tabular}{|l|c|}
\hline Autora & Citaciones \\
\hline Wood, E.J. & 211 \\
\hline Cohen, D.K. & 200 \\
\hline Baaz, M.E. & 125 \\
\hline Human Rights, Watch & 104 \\
\hline Brownmiller, S. & 87 \\
\hline Sivakumaran, S. & 84 \\
\hline Enloe, C. & 81 \\
\hline Johnson, K. & 80 \\
\hline
\end{tabular}

Fuente: Vosviewer a partir de información de Web of Science.

Al observar con detenimiento la información reportada por Web of Science, se identifica que por primera vez se reporta la información producida por una organización internacional de derechos humanos (Human Rights Watch). Esto resulta muy interesante si se tiene en cuenta que la producción científica en 
este campo, ha estado liderada por investigadores e investigadoras adscritos a la academia, mientras que las organizaciones no gubernamentales, tanto internacionales como nacionales, si bien han contribuido significativamente a la producción de conocimiento en torno a la violencia sexual, no ven reportada su información en este tipo de plataformas (gráfico 8).

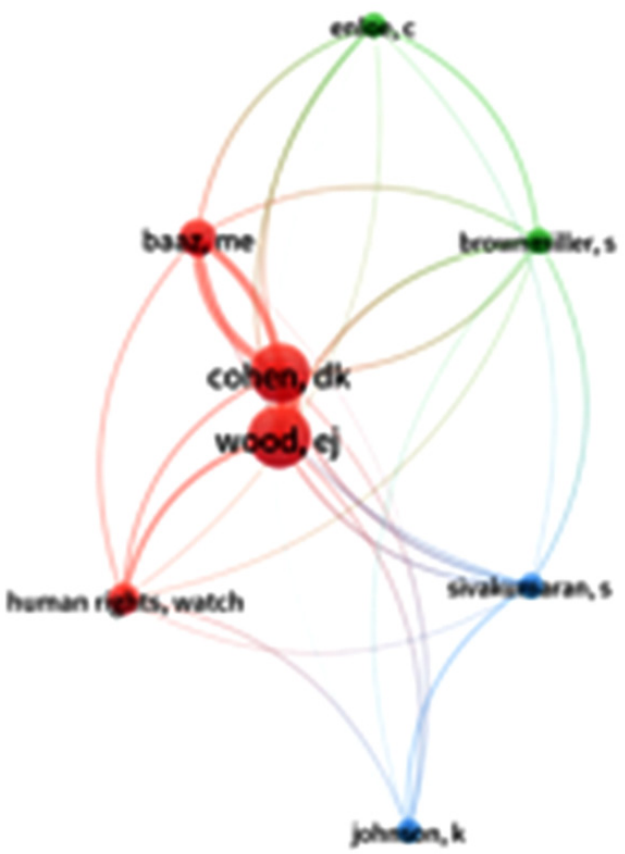

Gráfico 8. Cocitación Web of Science violencia sexual en general - mínimo de citación 80

Fuente: Vosviewer a partir de información de Web of Science

En relación con el caso colombiano (tabla 11), se identifica que son 10 las autoras con un umbral de citación igual o superior a 4, de acuerdo con Web of Science, encabezando la lista Wood con 11 citaciones, seguida de Cohen con 6 y el Centro Nacional de Memoria Histórica con 6, entre otros autores.

$\mathrm{Al}$ igual que en el punto anterior, se identifica que en el caso puntual de Colombia, son más las organizaciones sociales y estatales que son reportadas en Web of Science a diferencia de Scopus. Amnistía Internacional y el Centro Nacional de Memoria Histórica se encuentran dentro de los autores con más citaciones. No obstante, como se ve en la gráfica 9, contrario a lo ocurrido con Scopus y en la búsqueda general de Web of Science, la dispersión de citas entre autores es mucho mayor. 

en el conflicto armado, con énfasis en Colombia

Tabla 11. Autoras con umbral de citación de mínimo 80 en Web of Science - violencia sexual en Colombia.

\begin{tabular}{|l|c|}
\hline Autora & Citaciones \\
\hline Wood, E.J. & 11 \\
\hline Cohen, D.K. & 6 \\
\hline Centro Nacional de Memoria Histórica & 6 \\
\hline Gutiérrez-Sanín, Francisco & 5 \\
\hline Charlesworth, H. & 5 \\
\hline Lykes, M.B & 5 \\
\hline Meertens, D. & 4 \\
\hline Amnesty International (AI) & 4 \\
\hline Amnistía Internacional & 4 \\
\hline Clark, I.N. & 4
\end{tabular}

Fuente: Vosviewer a partir de información de Web of Science

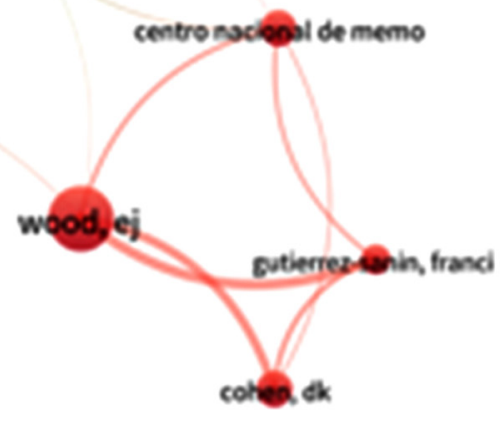

\section{Sviewer}

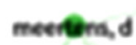

Gráfico 9. Cocitación Web of Science violencia sexual en Colombia - mínimo de citación 4 Fuente: Vosviewer a partir de información de Web of Science. 
Finalmente, en relación con los países con mayor productividad académica en materia de la violencia sexual en conflictos armados en general (tabla 7), se observa que en Scopus y Web of Science hay coincidencias interesantes que muestran como países líderes a Estados Unidos, el Reino Unido, Canadá, Australia y Alemania. En el caso de la violencia sexual en el conflicto armado en Colombia, en cambio, dicha producción es liderada por Estados Unidos seguido de Colombia, según Scopus, mientras que en Web of Science el primer lugar lo ocupa Colombia seguido de Estados Unidos.

\section{Análisis de contenido: temas de investigación}

En relación con el análisis del contenido de las publicaciones, se identificó como conveniente agrupar los resultados de Scopus y Web of Science para facilitar la consolidación y depuración de la información. Así, del total de 1442 resultados obtenidos para la violencia sexual en general, luego de fusionar mediante Zotero aquellos repetidos, se obtuvo una lista total de 1078 documentos. En el caso de los resultados para Colombia, del total de 100 documentos, luego de la fusión mediante Zotero se obtuvo un total de 72 textos.

Al hacer la revisión de la co-ocurrencia de las palabras clave sobre la violencia sexual en general con un umbral mínimo de 50 ocurrencias, se encontró que al procesar la información mediante Vosviewer, existen 22 términos que superan dicho umbral (tabla 12).

$\mathrm{Al}$ analizar las palabras clave con las cuales se asocia la violencia sexual en estos documentos (tabla 12 y gráfico 10) se encuentra que, por una parte, cobran relevancia otras formas de enunciación como la violación, las ofensas sexuales y los crímenes sexuales y, por otra, el perfil de actores relacionados con estos hechos también se pone de presente: mujeres, hombres, adultos, adolescentes, adultos jóvenes y niños. Aunque de la información extraída por esta vía no resulta claro si se asocian esos perfiles a las víctimas, pareciera coherente con la literatura dominante sobre el tema que ha centralizado a las víctimas en sus análisis.

La revisión de palabras clave concurrente para la violencia sexual en Colombia arrojó que de los 80 documentos, tienen concurrencia de 6 un total de 22 palabras (tabla 13).

Los resultados arrojaron que también son significativas aquellas palabras que se refieren a los perfiles de las personas involucradas (female, gender, adult, adolescent, male, child) y a los campos de conocimiento (human rights, psycology). Sin embargo, las formas de enunciación de la violencia sexual no son tan significativas y, en cambio, la noción de refugiado ocupa un lugar más central (gráfico 11). 

en el conflicto armado, con énfasis en Colombia

Tabla 12. Palabras clave con concurrencia mayor de 50 palabras.

\begin{tabular}{|l|c|}
\hline \multicolumn{1}{|c|}{ Keyword } & Occurrences \\
\hline human & 234 \\
\hline humans & 179 \\
\hline female & 181 \\
\hline war & 221 \\
\hline sexual violence & 316 \\
\hline article & 151 \\
\hline adult & 110 \\
\hline male & 109 \\
\hline rape & 157 \\
\hline violence & 148 \\
\hline sex offenses & 86 \\
\hline sexual crime & 89 \\
\hline adolescent & 75 \\
\hline psychology & 64 \\
\hline priority journal & 70 \\
\hline young adult & 55 \\
\hline posttraumatic stress disorder & 60 \\
\hline mental health & 54 \\
\hline child & 55 \\
\hline conflict & 75 \\
\hline human rights & 58 \\
\hline gender & 66 \\
\hline
\end{tabular}

Fuente: Vosviewer a partir de información de Scopus y Web of Science.

Estos resultados dan cuenta de vasos comunicantes entre la literatura general sobre la violencia sexual y aquella referida al caso colombiano; no obstante, podría pensarse que por la propia naturaleza del conflicto y contexto colombianos, la cuestión del refugio adquiere una mayor relevancia.

Finalmente, al analizar del total de 1078 documentos las palabras con concurrencia mayor a 10 (incluyendo palabras clave, título y resumen), encontramos 175 que superan dicho umbral (gráfico 12). 


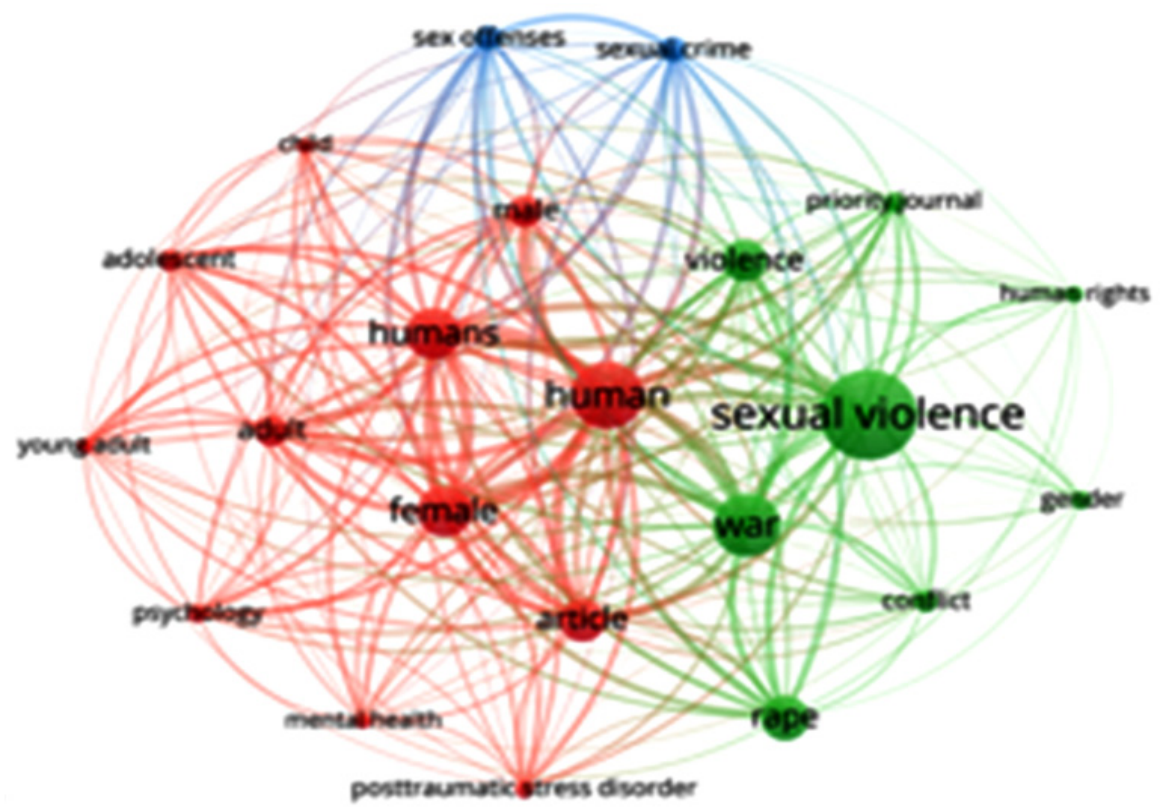

Gráfico 10. Coocurrencia de palabras clave - Violencia sexual en conflicto armado en general - Umbral mínimo de 50 palabras.

Fuente: Vosviewer a partir de la información de Scopus y Web of Science

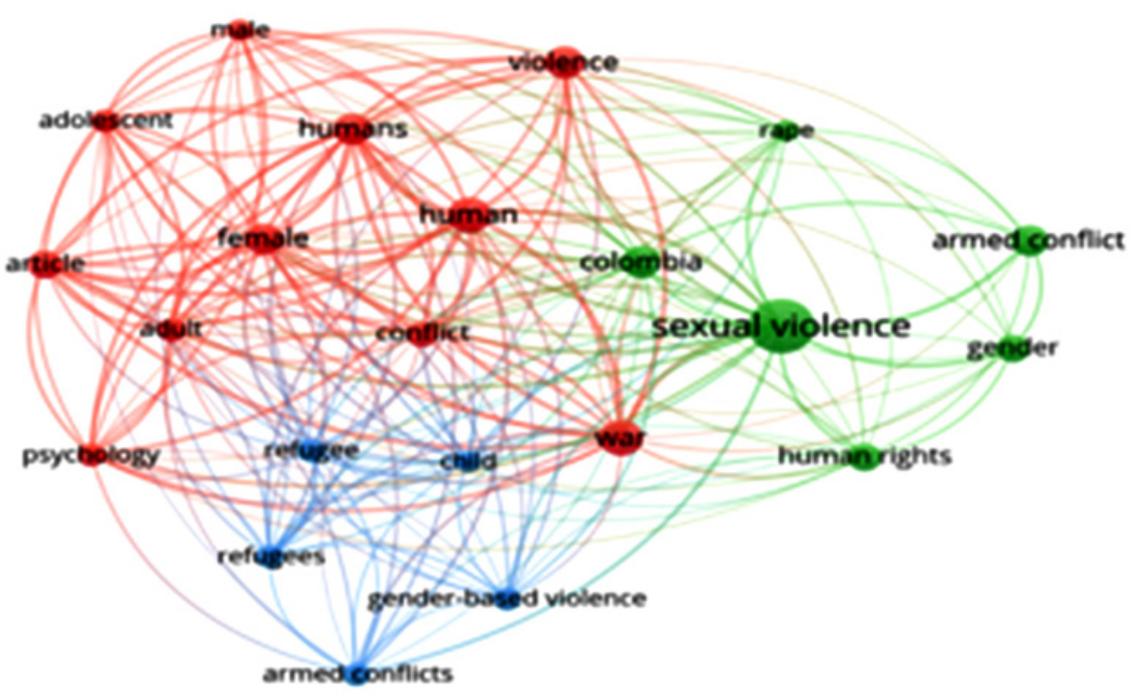

Gráfico 11. Coocurrencia de palabras clave - Violencia sexual en conflicto armado en Colombia - Umbral de 6 palabras

Fuente: Vosviewer a partir de la información de Scopus y Web of Science. 
Tabla 13. Palabras clave con concurrencia mayor de 6 palabras - violencia sexual en Colombia

\begin{tabular}{|l|c|}
\hline \multicolumn{1}{|c|}{ Kevword } & Occurrences v \\
\hline sexual violence & 40 \\
\hline human & 17 \\
\hline war & 17 \\
\hline violence & 14 \\
\hline Colombia & 14 \\
\hline female & 13 \\
\hline humans & 13 \\
\hline armed confHct & 13 \\
\hline article & 11 \\
\hline human rights & 10 \\
\hline gender & 10 \\
\hline conflict & 9 \\
\hline adult & 8 \\
\hline psychology & 7 \\
\hline adolescent & 7 \\
\hline refugee & 7 \\
\hline refugees & 7 \\
\hline armed conflicts & 7 \\
\hline gender-based violence & 7 \\
\hline male & 6 \\
\hline child & 6 \\
\hline rape & 6 \\
\hline
\end{tabular}

Fuente: Vosviewer a partir de información de Scopus y Web of Science.

De manera concordante con los resultados arrojados en palabras clave (gráfico 10), cuando miramos la concurrencia de palabras superior a 50 observamos para estos resultados (gráfico 11) que son las formas de enunciación sobre la violencia sexual (rape, sexual crime, sex offenses) y los perfiles de las personas involucradas (female, adult, male, adolescent, gender, child, young adult), los que ocupan un lugar central. Además, otros aspectos relacionados con los campos de estudio como psicología, desorden de estrés postraumático, salud mental y derechos humanos, se posicionan como elementos de análisis prioritarios. Al 


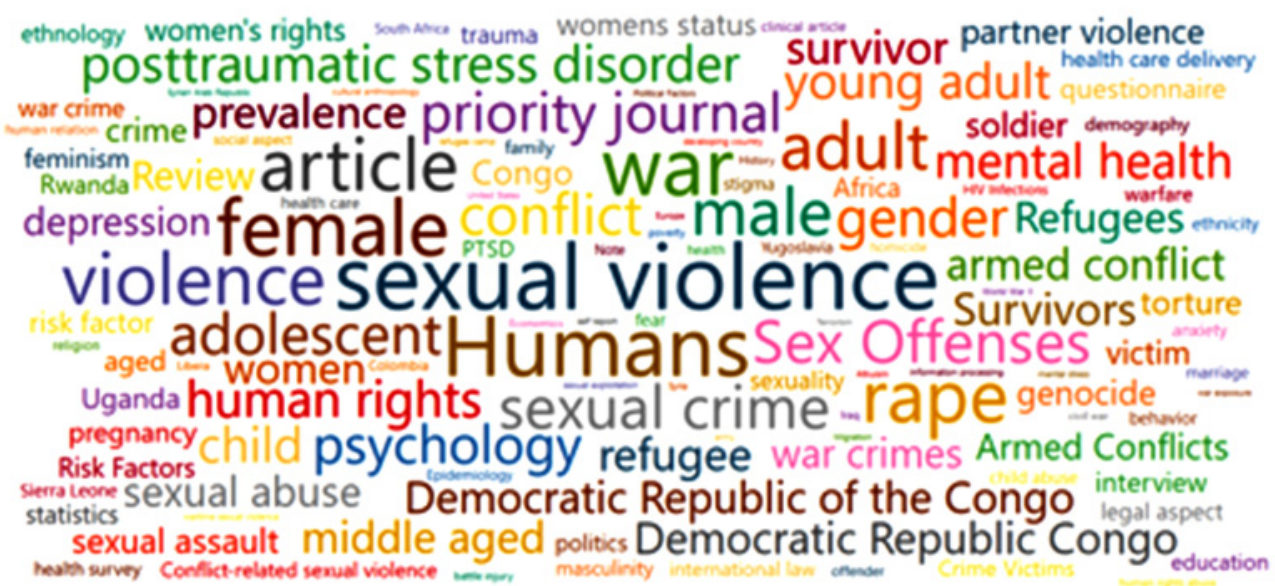

Gráfico 12. Resultados de palabras incluidas en palabras clave, título y resumen, con concurrencia mayor a 10

Fuente: Vantage Point con base en los resultados de Scopus y Web of Science

analizar las palabras concurrentes entre 40 y 50, encontramos que sobreviviente, refugiados, mujeres y República Democrática del Congo, son lugares comunes en los análisis.

Estas palabras contribuyen a identificar los aspectos sobre el fenómeno que están en debate o que sirven de soporte como lugares de enunciación del tema. Efectivamente, los resultados son concordantes con algunos de los temas de mayor debate: qué ingresa y no ingresa en la violencia sexual y cómo son los patrones de violencia; quiénes y cuáles son los perfiles de las partes involucradas y cuáles son los campos disciplinares más relevantes para su estudio.

\section{CONCLUSIÓN GENERAL}

La producción académica científica reportada en Scopus y Web of Science es concordante con la evolución del abordaje de la problemática de la violencia sexual en el conflicto armado en el campo del derecho internacional de los derechos humanos, en particular con la Agenda de Mujer, Seguridad y Paz impulsada por la ONU. En este sentido, aunque se observan publicaciones desde el año 1988, el ascenso continuo se observa desde el 2008, año en el cual el Consejo de Seguridad de la ONU asoció la violencia sexual como un asunto de paz y seguridad internacional.

En su mayoría las publicaciones reportadas por ambas plataformas se relacionan con el campo de las ciencias sociales y del derecho, siendo los principales 

en el conflicto armado, con énfasis en Colombia

documentos publicados los artículos en revistas académicas. Las revistas que más circulan sobre este campo están referidas a estudios feministas, asuntos humanitarios, cuestiones de justicia transicional, paz y ciencias de la salud.

Las autoras más prolíficas sobre el tema son mujeres, en su mayoría, quienes producen su conocimiento principalmente en países como Estados Unidos, Reino Unido, Canadá y Australia. Para el caso específico colombiano, la producción es mixta, tanto de autores individuales (hombres y mujeres) como institucionales tales como el Centro Nacional de Memoria Histórica y Amnistía Internacional. Esta diferencia resulta significativa, ya que evidencia un mayor involucramiento de instituciones gubernamentales y ONG en la producción académica en materia de la violencia sexual en el conflicto armado en Colombia.

En general llama la atención el mínimo número de organizaciones sociales cuya producción es registrada tanto en Scopus como en Web of Science, a pesar de ser entidades que contribuyen significativamente a la investigación de esta problemática. Esto puede obedecer a múltiples factores como el idioma, la forma como se registra y circula la información y el poco reconocimiento del aporte de las organizaciones sociales a la construcción del conocimiento. En cualquier caso, es un asunto a estudiar que requiere mayores análisis, puesto que pareciera que se trata de dos circuitos de conocimiento que no se reconocen ni se articulan, lo cual tiene efectos bidireccionales.

\section{REFERENCIAS}

Brilhante, A. V. M., Moreira, G. A. R., Vieira, L. J. E. de S., \& Catrib, A. M. F. (2016). A bibliometric study on gender violence. Saúde e Sociedade, 25, 703-715.

Comisión Interamericana de Derechos Humanos. (2006). Las mujeres frente a la violencia y la discriminación derivadas del conflicto armado en Colombia (OEA/Ser.L/V/II., Doc. 67).

Corte Penal Internacional. (2012). Situación en Colombia. Reporte Intermedio. https:/ / www.icc-cpi.int/NR/rdonlyres/3D3055BD-16E2-4C83-BA85-35BCFD2A7922/ 285202/OTP2012035032COLResumenEjecutivodelReporteIntermed.PDF

Engle, K. (2020). The Grip of Sexual Violence in Conflict: Feminist Interventions in International Law (Edición: 1). Stanford University Press.

Gutiérrez Sanín, F. (2014). El orangután con sacoleva. Debate.

Henry, N. (2014). The Fixation on Wartime Rape Feminist Critique and International Criminal Law. Social \& Legal Studies, 23(1), 93-111. https://doi.org/10.1177/ 0964663913499061 
Judith Velasco, Esther Arias, \& Mercedes Novo. (s. f.). Análisis bibliométrico sobre la investigación en violencia de género. Fundamentos y nuevas tendencias. https:/ / www.academia.edu/23586319/ANÁLISIS_BIBLIOMÉTRICO_SOBRE_LA_ INVESTIGACIÓN_EN_VIOLENCIA_DE_GÉNERO._FUNDAMENTOS_Y_ NUEVAS_TENDENCIAS

Office of Special Representative of the Secretary-General on Sexual Violence in Conflict. (2011). Progress report. https://www.un.org/sexualviolenceinconflict/wpcontent/uploads/report/team-of-experts-progress-report-2011/TOE-PROGRESSREPORT-2011.pdf

Palomo, J., Figueroa-Domecq, C., \& Laguna, P. (2017). Women, peace and security state-of-art: A bibliometric analysis in social sciences based on SCOPUS database. Scientometrics, 113(1), 123-148. https:// doi.org/10.1007/s11192-017-2484-x

Relatora Especial sobre la violencia contra la mujer, sus causas y consecuencias, ONU. (2002). Informe presentado por la Sra. Radhika Coomaraswamy, Relatora Especial sobre la violencia contra la mujer, sus causas y consecuencias: Misión a Colombia (1-7 de noviembre de 2001) (E/CN.4/2002/83/Add.3). http:/ / www.hchr.org.co/ documentoseinformes/documentos/html/informes/onu/resvm/E-CN-4-200283-Add3.html

Resolución 1325, Pub. L. No. S/RES/1325 (2000).

Resolución 1820, Pub. L. No. S/RES/1820 (2008).

Theidon, K. (2016). A greater measure of justice: Gender, violence and reparations. En E. Lewin \& L. M. Silverstein (Eds.), Mapping Feminist Anthropology in the TwentyFirst Century. Rutgers University Press. 
